\title{
Decimetric radio dot emissions
}

\author{
H. Mészárosová ${ }^{1,2}$, M. Karlický ${ }^{\text {, H. S. Sawant }}{ }^{1}$, F. C. R. Fernandes ${ }^{3}$, J. R. Cecatto ${ }^{1}$, and M. C. de Andrade $^{1}$ \\ 1 National Space Research Institute (INPE), São José dos Campos, SP, Brazil \\ 2 Astronomical Institute of the Academy of Sciences of the Czech Republic, 25165 Ondřejov, Czech Republic \\ e-mail: hana@asu.cas.cz \\ 3 Institute of Research and Development (IP\&D - UNIVAP), São José dos Campos, SP, Brazil
}

Received 30 April 2007 / Accepted 6 September 2008

\begin{abstract}
Context. We study a rare type of solar radio bursts called decimetric dot emissions.

Aims. In the period 1999-2001, 20 events of decimetric dot emissions observed by the Brazilian Solar Spectroscope (BSS) in the frequency range 950-2640 MHz are investigated statistically and compared with radio fine structures of zebras and fibers.

Methods. For the study of the spectral characteristics of the dot emissions we use specially developed Interactive Data Language (IDL) software called BSSView and basic statistical methods.

Results. We have found that the dm dot emissions, contrary to the fine structures of the type IV bursts (i.e. zebras, fibers, lace bursts, spikes), are not superimposed on any background burst emission. In the radio spectrum, in most cases the dot emissions form chains that appear to be arranged in zebra patterns or fibers. Because some zebras and fibers, especially those observed with high time and high spectral resolutions, also show emission dots (but superimposed on the background burst emission), we compared the spectral parameters of the dot emissions with the dots being the fine structure of zebras and fibers. For both these dots, similar spectral characteristics were found. Some similarities of the dot emissions can be found also with the lace bursts and spikes. For some events the dot emissions show structural evolution from patterns resembling fibers to patterns resembling zebras and vice versa, or they evolve into fully chaotic patterns.

Conclusions. For the first time, we present decimetric dot emissions that appear to be arranged in zebra patterns or fibers. We propose that these emissions are generated by the plasma emission mechanism at the locations in the solar atmosphere where the double resonance condition is fulfilled.
\end{abstract}

Key words. Sun: radio radiation - Sun: corona - Sun: flares

\section{Introduction}

Radio bursts and their fine structures give us diagnostic information about plasma processes in solar flares. One of the most important fine structures for such a diagnosis are zebras and fibers, which are considered to be the fine structures of type IV radio bursts (Slottje 1981; Isliker \& Benz 1994). The zebra pattern is observed as a set of nearly parallel light and dark stripes in the dynamic spectrum superposed on the broadband type IV burst continuum. There are many papers describing these fine structures as well as explaining them, see the review of Chernov (2006). At present, there is no universally accepted interpretation of these types of fine structures. The most commonly used theoretical models of these structures are: a) those based on double plasma resonance (Zheleznyakov \& Zlotnik 1975; Ledenev et al. 2001); and b) based on the interaction between plasma waves and whistlers (Kuijpers 1975; Chernov 2006). For further models, see LaBelle et al. (2003).

An increase of the frequency and time resolution of new radiospectrographs reveals that there are even sub-structures of these fine structures. For example, Chernov et al. (2003, 2005) have reported zebras with spikes. Based on these observations they have included coupling of ion-sound waves with whistlers into their model. Similar sub-structures of zebras have been presented by Kuznetsov \& Tsap (2007) and Zlobec \& Karlický (2007). Jiřička et al. (2001) have found examples of decimetric fibers with dots as well as examples of bursts with rapid frequency variations (lace bursts). Due to similarities between the lace bursts and zebra pattern lines a model based on the double resonance mechanism was suggested for both these fine structures (Karlický et al. 2001). A similar model, but with the plasma turbulence in the radio source was proposed for the narrow band dm-spikes (Bárta \& Karlický 2001).

Besides all these fine structures mentioned above, new observations with high time and spectral resolution revealed very rare bursts named by Sawant et al. (2002) and Krishan et al. (2003) as $\mathrm{dm}$ dot emissions.

In this paper we study these dot emissions in detail. The layout of the paper is as follows. In Sect. 2 we outline the instrument and observational data. Then we analyze their spectral characteristics and compare them mainly with the fine structure of zebras and fibers (Sect. 3). Discussion of results and conclusions are given in Sect. 4.

\section{Instrument and observational data}

The frequency range of the BSS is $950-2640 \mathrm{MHz}$ with variable time and frequency resolution between 10-1000 ms and 1-10 MHz, respectively (Sawant et al. 2001). The BSS has observed 20 events (Table A.1) with 62 groups of dot emissions during period 1999-2001. Most of these events (15 of 20) were measured with a time and frequency resolution of about $50 \mathrm{~ms}$ and 5 or $10 \mathrm{MHz}$, respectively. Thus, the high sensitivity, time 
and frequency resolution of the BSS has led to the observation of individual, discrete dots. With lower resolutions, the chain of dots would appear to be a line.

Of the 20 events listed in Table A.1 (containing dot emissions) there are 19 events associated with GOES X-ray flares. For 6 of 18 GOES flares (March 19, 1999, October 19, 1999, September 20, 2000, September 25, 2000, April 26, 2001 and September 11, 2001) the dot emissions occur in the impulsive phase; for 13 of 18 GOES flares they occur in the declining phase of the associated flare.

\section{Statistics and analysis of data}

The dot emissions are a very rare type of solar radio burst. They are characterized by their dot form and, contrary to the fine structures of the type IV bursts (i.e. zebras, fibers, lace bursts, spikes), they are not superimposed on any background burst emission see the following examples. In many cases these dot emissions were arranged into chains. According to the appearance of these chains on the dynamic spectrum we divided the chains into two types: a) zebra-like chains; and b) fiber-like chains. Thus we found that in 26 groups of dot emissions the chains appeared to be arranged as zebras and in 36 groups they appeared to be arranged as a group of fibers. In other cases these chains had a more chaotic structure resembling the narrow-band spikes or even lace bursts.

\subsection{Dot-emissions which appear to be arranged as zebras and fibers (with no background burst emission)}

Using the BSS we have observed 8 events (Nos. 1, 6, 10, 11, 13 and 17-19, Table A.1) with 18 groups of dot-emission chains arranged as a zebra pattern and 13 events (Nos. 1, 3-6, 10, 12-15 and 17-19, Table A.1) with 19 groups of dot-emission chains arranged as fiber bursts. These events are associated with rather weak X-ray GOES flares (B4.0-C5.9, Table A.1).

Let us define the fiber-like and zebra-like chains of the dot emissions. In the fiber-like chains, the dot emissions are concentrated along rather highly drifting chains. On the other hand, in the case of zebra-like chains, the dot emissions are arranged in rather horizontal or wave-like chains. This means that in one zebra-like chain the dot emissions are observed nearly at the same frequency.

An example of a group of dot emissions arranged as zebralike chains observed on March 19, 1999 is shown at Fig. 1. Panel a shows soft X-ray fluxes $1.0-8.0 \AA$ and $0.5-4.0 \AA$ observed by GOES 10. Panel b presents a global view of the dot emissions. The time and frequency resolution is $100 \mathrm{~ms}$ and $5 \mathrm{MHz}$, respectively. Panel c reveals a detailed view showing the dot emissions with undulating chains resembling the zebra pattern. Panel d shows the radio flux in time at $1400 \mathrm{MHz}$ where peaks with an intensity of $\sim 130$ SFU correspond to individual dot emissions (horizontal line in panel c). Panel e shows the radio flux depending on frequency observed at 15:25:53.7 UT (vertical line in panel c). The peak with an intensity of 134 SFU corresponds to the individual dot-emission at $1400 \mathrm{MHz}$.

An example of groups of dot emissions arranged as fiber-like chains observed on October 19, 1999 is given in Fig. 2. Panel a shows soft X-ray fluxes observed by GOES 8 . Panel b presents a global view of the dot emissions. The time and frequency resolution is $55 \mathrm{~ms}$ and $5 \mathrm{MHz}$, respectively. Panel c shows a detailed view of the dot emissions arranged as a group of positively drifting fiber-like chains. The average frequency drift of individual

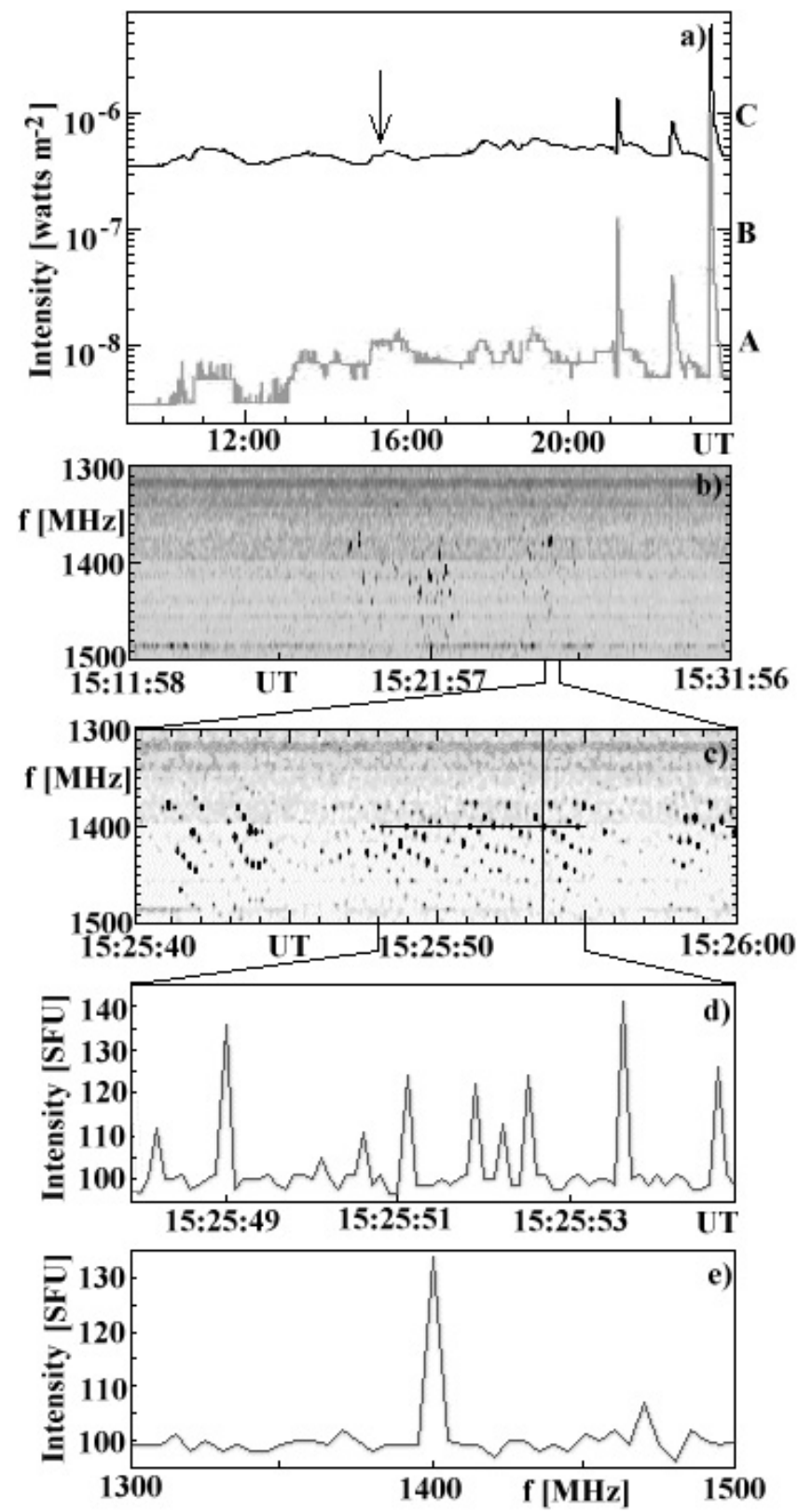

Fig. 1. Dot-emissions (in black) arranged as zebra-like chains observed on March 19, 1999: a) soft X-ray fluxes 1.0-8.0 Å (black) and 0.5-4.0 $\AA$ (grey) observed by GOES 10 (arrow shows the time of dot emissions observation), b) global view of dot emissions, c) zebra-like chains, d) radio flux in time at $1400 \mathrm{MHz}$ (horizontal line in c), e) radio flux in frequency observed at 15:25:53.7 UT (vertical line in c).

fibers is $+350 \mathrm{MHz} \mathrm{s}^{-1}$. Panel d presents the radio flux in time at $1265 \mathrm{MHz}$ where the peaks with intensity >200 SFU correspond to individual dot emissions (horizontal line, panel c). Panel e) shows the radio flux as a function of frequency observed at 17:03:53.3 UT (vertical line, panel c). The peaks with intensity $>200$ SFU correspond to the individual dots. In both cases (Figs. 1, 2), the intensity of the quiet sun level is $\sim 100 \mathrm{SFU}$ (panels $\mathrm{d}, \mathrm{e})$. Thus, there is no background burst emission.

We studied some of characteristic parameters (average values) of the dot emissions for (i) 18 groups of zebra-like chains and (ii) 49 selected characteristic individual fiber-like chains. In the first case the frequency separation between neighboring zebra-like chains measured at one specific time is $66 \mathrm{MHz}$ 

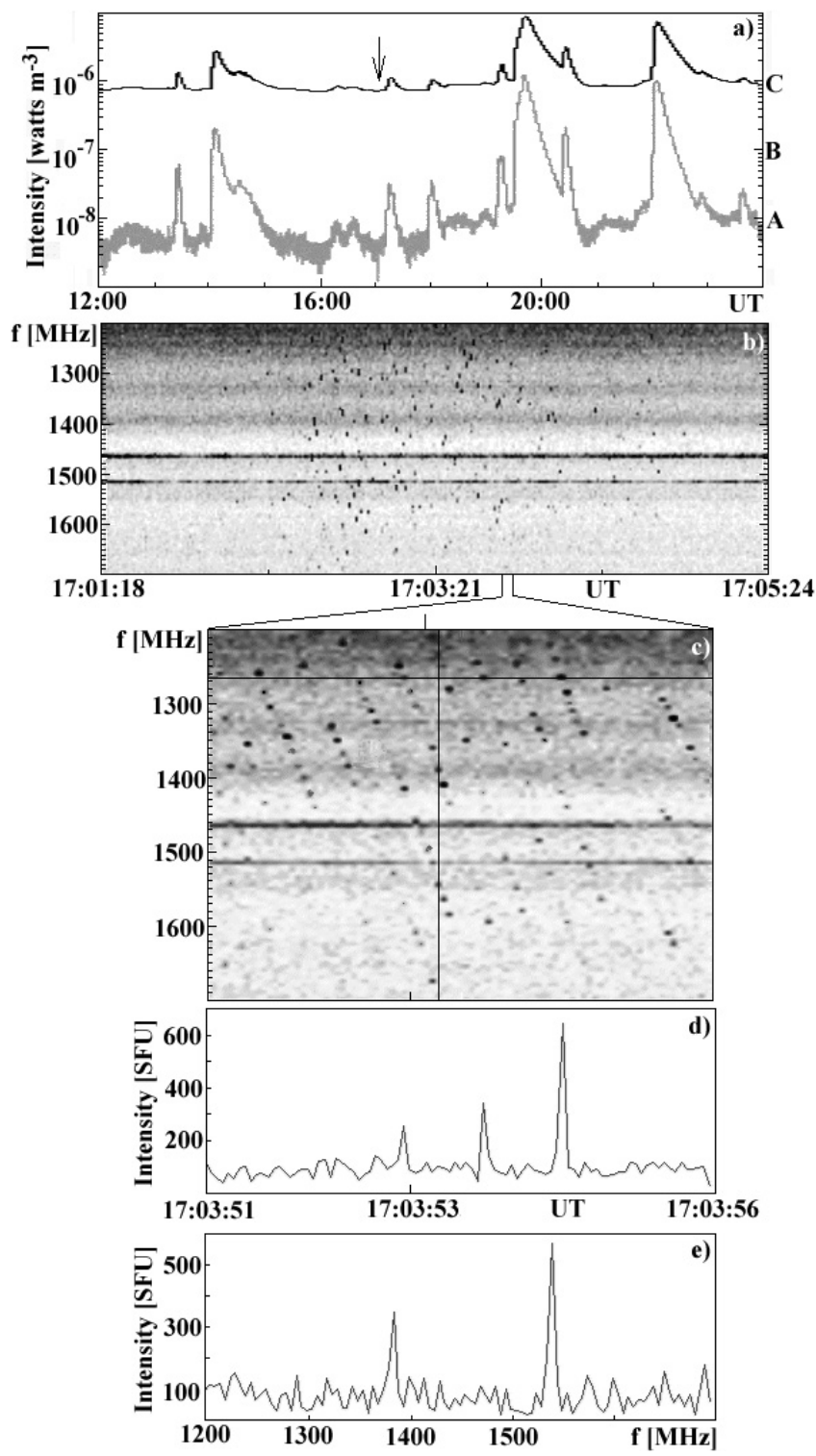

Fig. 2. Dot-emissions (in black) arranged as fiber-like chains observed on October 19, 1999: a) soft X-ray fluxes 1.0-8.0 $\AA$ (black) and 0.5-4.0 $\AA$ (grey) observed by GOES 8 (arrow shows the time of dot emissions observation), b) global view of dot emissions, c) fiber-like chains, d) radio flux in time at $1265 \mathrm{MHz}$ (horizontal line in c). e) radio flux in frequency observed at 17:03:53.3 UT (vertical line in c).

and the time separation between successive dot emissions in the same zebra-like chain is $107 \mathrm{~ms}$. In the second case the time separation between neighboring fiber-like chains measured at one specific frequency is $485 \mathrm{~ms}$ and the frequency separation between succeeding dots in the same fiber-like chain is $14 \mathrm{MHz}$. The individual fiber-like chains show both negative and positive frequency drift (+415 $\mathrm{MHz} \mathrm{s}^{-1}$ and $\left.-522 \mathrm{MHz} \mathrm{s}^{-1}\right)$.

The radio spectra in our data set show the dot emissions arranged in chains as well as those with very chaotic distributions. We have found examples showing that both zebra-like chains and fiber-like chains evolve from one to the other. In some cases, the beginning or ending parts of zebra-like chains are very similar to negatively or positively drifting fiber-like chains (for example the beginning of the zebra-like chains in Fig. 1 in comparison to the fiber-like chains in Fig. 2). Some similarities in morphology of the dot emissions in their frequency spectrum can
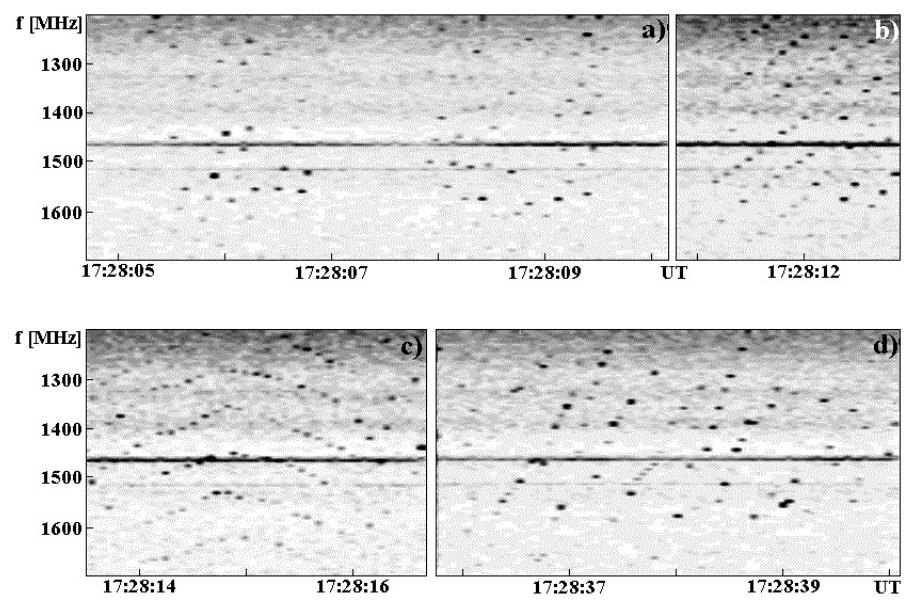

Fig. 3. Example of the arrangement of series of the dot emissions (in black) observed on October 19, 1999: irregular dot emissions (panel a)), fiber-like chains (panel b)), zebra-like chains (panel c)), and irregular dot emissions (panel d)).

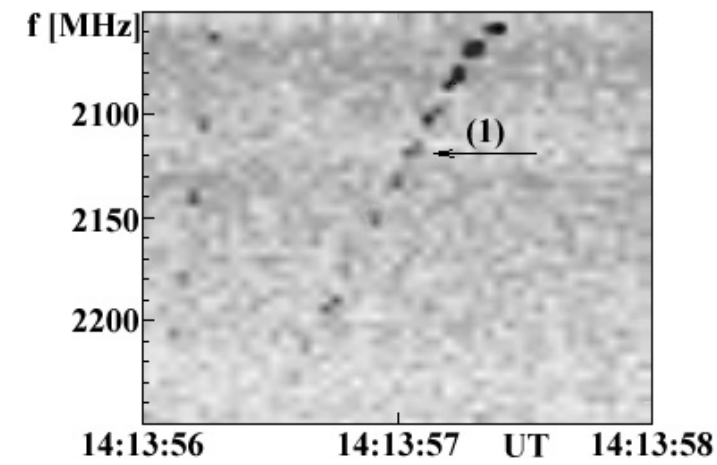

Fig. 4. Fiber-like chain (1) observed on September 11, 2001.

Table 1. Ratios of the frequencies of successive individual dot emissions in the selected fiber-like chain (1) observed on September 11, 2001 (Fig. 4).

\begin{tabular}{lcccccc}
\hline \hline$f[\mathrm{MHz}]$ & 2086 & 2101 & 2118 & 2135 & 2154 & 2194 \\
Ratio & 1.007 & 1.007 & 1.008 & 1.008 & 1.009 & 1.019 \\
\hline
\end{tabular}

be found even with the lace bursts and spikes (see Figs. 1, 2, panels b). Figure 3 shows an example of structural evolution of the group of the dot emissions observed on October 19, 1999. This group starts with some irregular dot emissions (panel a) but later they evolve into almost regular fiber-like (panel b) and zebra-like (panel c) chains. At the end the group of dot emissions again become irregular (panel d). Table 1 shows the ratios of succeeding dot-emission frequencies in the selected fiber-like chain (1) observed on September 11, 2001 (Fig. 4). The tendency of this ratio to increase with frequency increasing can be seen here.

\subsection{Fine structure of zebras and fibers - dots}

For comparison with the studied dot emissions we analyzed in detail the dots which sometimes appear on the radio spectra with high-time and frequency resolutions as fine structures of zebras and fibers. Using the BSS we observed 2 events (Nos. 7 and 8, Table A.1) with 8 zebra patterns and 5 events (Nos. 2, 7 , 9, 16 and 20, Table A.1) with 17 groups of fiber bursts. These events are associated with rather strong X-ray GOES flares (C3.3-X2.3, Table A.1). Contrary to the dot emissions described 
in Sect. 3.1 these dots are the fine structure of zebras and fibers. The zebra pattern and fiber bursts consist of intermittent emission (bright) and absorption (dark) stripes superimposed on the background type IV burst. These emission stripes show distinguishable (isolated) individual dots. Individual emission stripes have an intensity higher than the intensity of the background type IV burst but lower then the intensity of the dots.

An example of the dots as the fine structure of the zebra pattern observed on June 6, 2000 is given in Fig. 5. Panel a) shows soft X-ray fluxes observed by GOES 10 . The global view of the zebra pattern (panel b) reveals rather horizontal and wave-like emission (bright) and absorption (dark) stripes. The time and frequency resolution is $54 \mathrm{~ms}$ and $5 \mathrm{MHz}$, respectively. Panel c) shows a detailed view with distinguishable individual dots superimposed on zebra stripes. In panel d) the radio flux in time at $1375 \mathrm{MHz}$ is shown where the highest peaks (>400 SFU) correspond to individual dots (the horizontal line in panel c). Panel e) shows the radio flux as a function of frequency at 15:54:44.6 UT (the vertical line in panel c) where the highest peaks (>400 SFU) correspond to individual dots. These peaks (panels d, e) are superimposed on the background burst emission with the intensity $\sim 300$ SFU.

An example of dots as the fine structure of fibers observed on September 9, 1999 is shown in Fig. 6. Panel a) shows soft X-ray fluxes observed by GOES 10. A global view (panel b) of a group of fibers (bright) shows fibers with similar frequency drift. The time and frequency resolution is $58 \mathrm{~ms}$ and $5 \mathrm{MHz}$, respectively. Panel c) reveals a detailed view of individual fibers with distinguishable dots. The average frequency drift of individual fibers is $-60 \mathrm{MHz} \mathrm{s}^{-1}$. In panel d) the radio flux in time at $2500 \mathrm{MHz}$ is shown. The highest peaks ( $>1400 \mathrm{SFU}$ ) correspond to individual dots (the horizontal line in panel c). Panel e) shows the radio flux as a function of frequency at 17:05:46.5 UT (the vertical line in panel c) where the highest peaks (>1400 SFU) correspond to individual dots. These peaks (panels $\mathrm{d}$, e) are superimposed on the background burst emission with an intensity of $\sim 1200$ SFU. In both cases (Figs. 5, 6), the intensity of the quiet sun level is $\sim 100 \mathrm{SFU}$.

We investigated some characteristic parameters (average values) of the dots as fine structure for (i) 8 zebras and (ii) 40 selected characteristic individual fibers (of 17 groups). In the first case the frequency separation between individual zebra emission stripes measured at one specific time is $18 \mathrm{MHz}$ and the time separation between successive dots in the same zebra emission stripe is $125 \mathrm{~ms}$. In the second case the time separation between neighboring fibers in a group measured at one specific frequency is $472 \mathrm{~ms}$ and the frequency separation between succeeding dots in the same fiber is $8 \mathrm{MHz}$. We have found that individual fibers have only negative frequency drift $\left(-61 \mathrm{MHz} \mathrm{s}^{-1}\right)$.

\section{Discussion and conclusions}

Twenty events of decimetric dot emissions were observed during the period 1999-2001 in the frequency range 950-2600 MHz using the BSS (Table A.1). We have found that the dm dot emissions, contrary to the fine structures of the type IV bursts (i.e. zebras, fibers, lace bursts, spikes), are not superimposed on any background burst emission. In the radio spectrum, in most cases the dot emissions form chains similar to zebras or fibers. On the other hand, some zebras and fibers, especially those observed with high-time and high-spectral resolutions, show also emission dots but they are superimposed on the burst emission.

We have found that average values of some parameters are similar for both the zebra/fiber-like chains and zebra/fibers: the
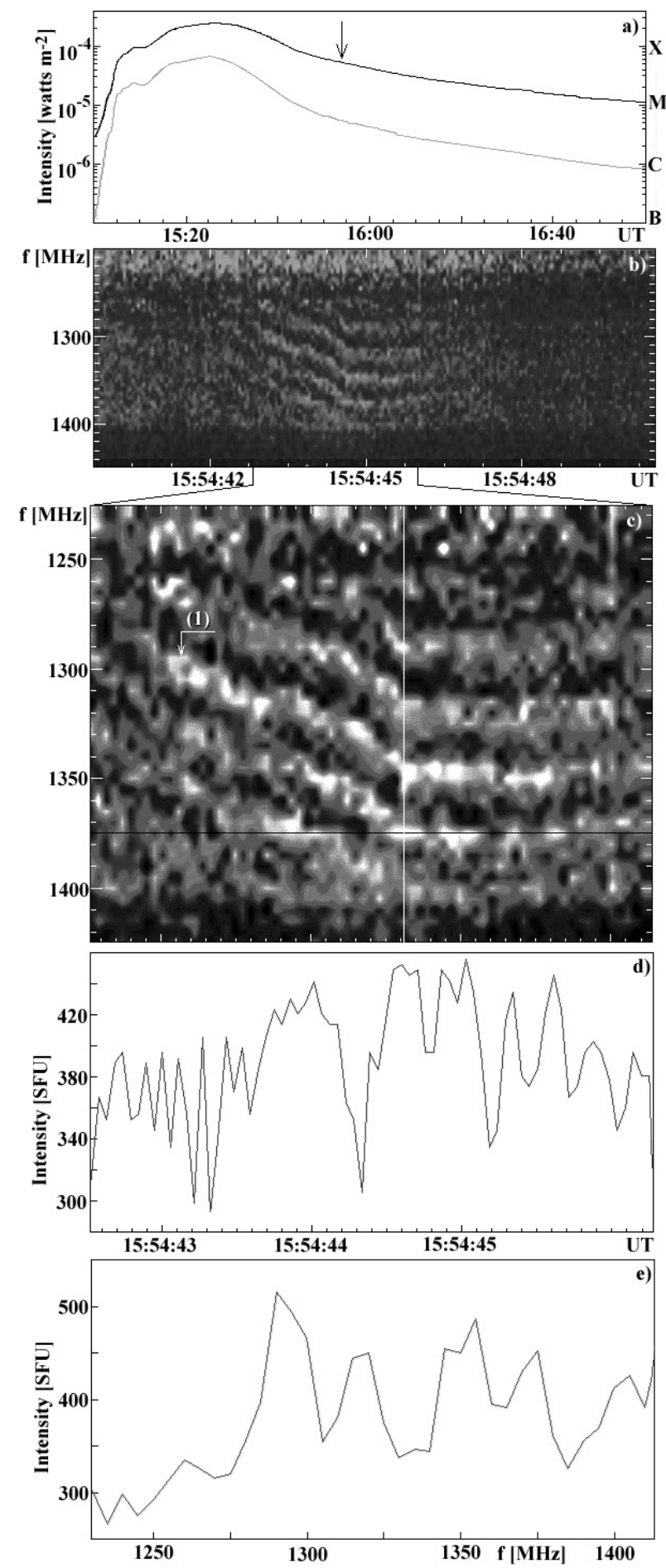

Fig. 5. Fine structure (dots) of the zebra observed on June 6, 2000: a) soft X-ray fluxes $1.0-8.0 \AA$ (black) and $0.5-4.0 \AA$ (grey) observed by GOES 10 (arrow shows the time of zebra observation), b) global view of a zebra, c) individual dots of a zebra, d) radio flux in time at $1375 \mathrm{MHz}$ (horizontal line in c), e) radio flux in frequency at 15:54:44.6 UT (vertical line in c).

time separation between fiber-like chains ( $485 \mathrm{~ms}$ ) and between individual fibers $(472 \mathrm{~ms}$ ), the average frequency separation between succeeding dots in the same fiber-like chain $(14 \mathrm{MHz})$ and fibers $(8 \mathrm{MHz})$, the average time separation between succeeding dots in the same zebra-like chain $(107 \mathrm{~ms})$ and zebra emission stripe (125 ms). We have also studied the average time 


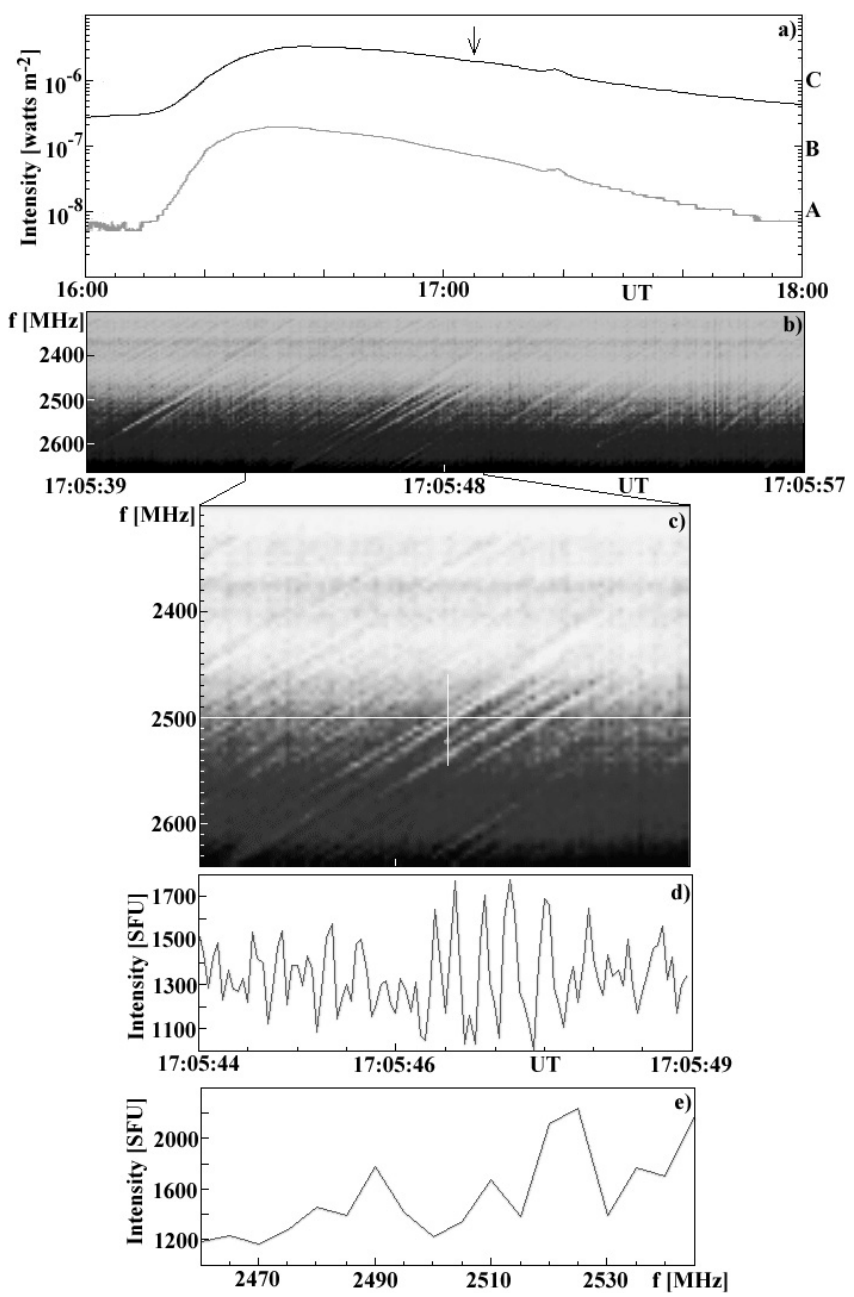

Fig. 6. Fine structure (dots) of the fibers observed on September 9, 1999: a) soft X-ray fluxes 1.0-8.0 $\AA$ (black) and 0.5-4.0 $\AA$ (grey) observed by GOES 10 (arrow shows time of fiber observation), b) global view of fibers (bright), c) individual dots of fibers, d) radio flux in time at $2500 \mathrm{MHz}$ (horizontal line in c), e) radio flux in frequency at 17:05:46.5 UT (vertical line in c).

separation between succeeding dots of fiber-like chains (Fig. 2, panel c) close to $1265 \mathrm{MHz}$ (on average $422 \mathrm{~ms}$ ) and between succeeding fiber dots (Fig. 6, panel d) at $2500 \mathrm{MHz}$ (on average $348 \mathrm{~ms}$ ). Thus, zebra-like chains as well as zebra emission stripes have significantly shorter time intervals between succeeding dots than fiber-like chains and fibers since zebra patterns are concentrated in horizontal structures. Some similarities of the dot emissions also can be found with the lace bursts and spikes (see Figs. 1, 2, panels b). For some events the dot emissions show structural evolution from the patters resembling fibers to the patterns resembling zebras and vice versa, or they evolve into fully chaotic patterns (Fig. 3).

Considering all aspects of the observed dot emissions, we think that the dot emissions are generated in a similar way as zebras (Ledenev et al. 2001) or lace bursts (Karlický et al. 2001). Thus, we propose that the dot emissions are produced in the solar atmosphere at the locations where the so-called double resonance condition is fulfilled: $\omega_{\mathrm{UH}}=\left(\omega_{\mathrm{pe}}^{2}+\omega_{\mathrm{Be}}^{2}\right)^{1 / 2}=s \omega_{\mathrm{Be}}$, where $\omega_{\mathrm{UH}}, \omega_{\mathrm{pe}}$, and $\omega_{\mathrm{Be}}$ are the upper hybrid, electron plasma and cyclotron frequencies, and $s$ is the integer harmonic number. The upper-hybrid waves at these locations can be generated e.g. by the anisotropic beam $\left(T_{\perp}>T_{\|}\right.$, where $T_{\perp}$ and $T_{\|}$are the temperatures of energetic electrons across and along the magnetic field, respectively) accelerated during the flare primaryenergy processes. The beam anisotropy can be naturally formed along magnetic field lines by an escape of fast electrons from slower ones. Then these upper-hybrid waves are transformed to electromagnetic waves (with the frequency $\omega_{\mathrm{el}} \approx \omega_{\mathrm{UH}}$ or $\left.\omega_{\mathrm{el}} \approx 2 \omega_{\mathrm{UH}}\right)$, which are observed by radiospectrographs on the Earth. This process is a resonant one which means that its intensity can be several orders of magnitude higher than those associated with non-resonant processes. This can explain the fact that at times of dot emission no background burst emission was detected.

Using this model we can explain not only individual dot emissions, but also their chains. The beam along its trajectory generates dot emissions in several resonance locations ( $s$-harmonics). The higher $s$ means a higher height of the dotemission source in the solar atmosphere. Thus fiber-like chains of the dot emissions can be formed. On the other hand, zebralike chains of the dot emissions can be explained by a sequence of anisotropic beams producing dot emissions nearly at the same position on the same $s$-harmonic. Even the whistler waves, which are considered in the model of fibers, can enhance emission at the locations with double resonance conditions. Therefore both forms of zebra-like and fiber-like chains are possible.

There is also another important aspect of the model. It can explain the tendency that the frequency ratio of neighboring dot emissions on the same fiber-like chain increases with increasing frequency as shown in Table 1. Using the resonance condition for the $s$-harmonic frequency we can write

$\omega_{s}=\frac{\omega_{\text {pes }}}{\left(1-\frac{1}{s^{2}}\right)^{1 / 2}}$

where $\omega_{\text {pes }}$ is the electron plasma frequency at the $s$-resonance. Thus, the ratio of the neighboring dot frequencies is

$\frac{\omega_{s}}{\omega_{s+1}}=\frac{\omega_{\text {pes }}}{\omega_{\text {pes }+1}}\left[\frac{s^{3}(s+2)}{(s+1)^{3}(s-1)}\right]^{1 / 2}$.

If we take roughly $\omega_{\text {pes }} \approx \omega_{\text {pes+1 }}$ (it is assumed that the density decreases with height much more slowly than the magnetic field) then $\omega_{2} / \omega_{3} \approx 1.09, \omega_{3} / \omega_{4} \approx 1.03, \omega_{4} / \omega_{5} \approx 1.01$, and so on. These values show the same trend as those presented in Table 1.

Due to similarities in morphology and characteristic properties between the dot emissions and dots in fine structures of zebras and fibers, we think that both have a similar physical origin. We found that fiber-like chains of the dot emissions evolve into zebra-like chains and vice versa. These changes are in agreement with the idea of Chernov et al. (1998) who proposed that both the zebras and fibers are generated by whistler packets. The propagating whistler packets may also generate dot emissions at the positions in the solar atmosphere where the double resonant conditions are fulfilled (see also, Sawant et al. 2002; \& Krishan et al. 2003). Furthermore, we propose that the chaotic character of some groups of the dot emissions is due to rapidly varying plasma parameters (in the MHD turbulence) in the region of the dot-emission sources.

Acknowledgements. The authors thank the anonymous referee and Prof. P. Janardhan for their useful remarks that improved this paper. H.M. acknowledges the FAPESP support for the project 2006/50039-7. M.K. acknowledges the support from the Grant IAA300030701 of the Academy of Sciences of the Czech Republic. F.C.R.F. thanks CNPq for scholarship (proc. 310005/2005-1). J.R.C. acknowledges the CNPq support for project 475723/2004-0. 
Appendix A:

Table A.1. Basic characteristics of the events with studied groups of dot emissions.

\begin{tabular}{|c|c|c|c|c|c|c|c|c|c|c|c|c|c|}
\hline \multirow[b]{2}{*}{ No. } & \multirow[b]{2}{*}{ Flare } & \multicolumn{2}{|c|}{ Radio } & \multicolumn{4}{|c|}{ GOES } & \multirow[b]{2}{*}{$\begin{array}{l}\text { Start } \\
\text { [UT] } \\
\end{array}$} & \multirow[b]{2}{*}{$\begin{array}{l}\text { Max } \\
\text { [UT] }\end{array}$} & \multirow[b]{2}{*}{$\begin{array}{l}\text { End } \\
\text { [UT] }\end{array}$} & \multirow{2}{*}{$\begin{array}{l}\mathrm{H}_{\alpha} \\
\text { Optic. } \\
\text { Imp. }\end{array}$} & \multirow[b]{2}{*}{ Position } & \multirow[b]{2}{*}{$\begin{array}{c}\text { NOAA } \\
\text { AR \# }\end{array}$} \\
\hline & & $\begin{array}{l}\text { Start } \\
\text { [UT] }\end{array}$ & $\begin{array}{l}\text { End } \\
\text { [UT] }\end{array}$ & $\begin{array}{l}\text { Start } \\
\text { [UT] }\end{array}$ & $\begin{array}{l}\text { Max } \\
\text { [UT] }\end{array}$ & $\begin{array}{l}\text { End } \\
{[\text { [UT] }}\end{array}$ & $\begin{array}{l}\text { X-ray } \\
\text { Imp. }\end{array}$ & & & & & & \\
\hline 1 & Mar. 19, 1999 & $15: 19$ & $15: 27$ & $14: 55$ & $15: 29$ & $16: 15$ & $\mathrm{~B} 4.0$ & & & & & & \\
\hline 2 & Sep. 09, 1999 & $17: 05$ & $17: 15$ & $16: 13$ & $16: 37$ & $17: 08$ & C3.3 & $16: 16$ & $16: 29$ & $17: 47$ & SF & N13 E37 & 8690 \\
\hline 3 & Sep. 13, 1999 & $17: 00$ & 17:01 & $16: 30$ & $16: 45$ & 17:04 & $\mathrm{C} 2.6$ & $16: 38$ & $16: 44$ & $17: 59$ & $1 \mathrm{~N}$ & N15 E06 & 8693 \\
\hline 4 & Oct. 07,1999 & $15: 53$ & $15: 54$ & $10: 08$ & $13: 44$ & $17: 34$ & C4.2 & & & & & & \\
\hline 5 & Oct. 18,1999 & $16: 26$ & $16: 27$ & $15: 57$ & $16: 07$ & $16: 28$ & $\mathrm{C} 1.2$ & & & & & & \\
\hline 6 & Oct. 19, 1999 & $17: 02$ & $17: 29$ & $17: 07$ & $17: 16$ & $17: 21$ & $\mathrm{C} 1.1$ & $17: 12$ & $17: 14$ & $17: 22$ & SF & S15 E19 & 8737 \\
\hline 7 & Jun. 06, 2000 & $15: 18$ & $16: 56$ & $14: 58$ & $15: 25$ & $15: 40$ & $\mathrm{X} 2.3$ & $15: 03$ & $15: 28$ & $15: 40$ & $2 \mathrm{~B}$ & N20 E13 & 9026 \\
\hline 8 & Jul. 11, 2000 & $13: 46$ & $13: 47$ & $12: 12$ & $13: 10$ & $13: 35$ & $\mathrm{X} 1.0$ & $13: 20$ & $13: 23$ & $18: 37$ & $2 \mathrm{~N}$ & N18 E27 & 9077 \\
\hline 9 & Jul. 14, 2000 & $12: 53$ & $12: 54$ & \multicolumn{2}{|c|}{ no data } & & & $12: 54$ & $12: 56$ & $13: 17$ & SF & S09 W01 & 9002 \\
\hline 10 & Sep. 18,2000 & $13: 59$ & 14:01 & $12: 12$ & $12: 24$ & 14:04 & C3.3 & $13: 52$ & $13: 52$ & $13: 56$ & SF & N14 E65 & 9167 \\
\hline 11 & Sep. 19, 2000 & $15: 25$ & $15: 27$ & $15: 13$ & $15: 19$ & $15: 24$ & $\mathrm{C} 3.0$ & $15: 16$ & $15: 17$ & $15: 38$ & $1 \mathrm{~F}$ & S08 E12 & 9166 \\
\hline 12 & Sep. 20, 2000 & $15: 27$ & $15: 28$ & $15: 19$ & $15: 31$ & $15: 42$ & $\mathrm{C} 3.1$ & $15: 14$ & $15: 21$ & $15: 45$ & SF & N09 E41 & 9169 \\
\hline 13 & Sep. 22, 2000 & $15: 17$ & $15: 18$ & $12: 40$ & $13: 19$ & $15: 18$ & C5.9 & & & & & & \\
\hline 14 & Sep. 25,2000 & $15: 20$ & $15: 22$ & $15: 18$ & $15: 35$ & $16: 39$ & $\mathrm{C} 1.1$ & & & & & & \\
\hline 15 & Sep. 29, 2000 & $16: 23$ & $16: 35$ & $16: 12$ & $16: 15$ & $16: 33$ & $\mathrm{C} 1.4$ & & & & & & \\
\hline 16 & Apr. 26, 2001 & $12: 57$ & $12: 58$ & $11: 26$ & $13: 12$ & $13: 19$ & M7.8 & $12: 11$ & $13: 11$ & $14: 31$ & $2 \mathrm{~B}$ & N17 W31 & 9433 \\
\hline 17 & Aug. 23, 2001 & $17: 32$ & $17: 33$ & $16: 57$ & $17: 04$ & $17: 48$ & $\mathrm{C} 2.1$ & & & & & & \\
\hline 18 & Sep. 11, 2001 & $14: 13$ & $14: 18$ & 14:00 & 14:37 & $15: 08$ & C3.2 & $14: 16$ & $14: 39$ & $15: 30$ & $\mathrm{SF}$ & N13 E35 & 9615 \\
\hline 19 & Sep 20, 2001 & $13: 34$ & $13: 36$ & $12: 54$ & 13:03 & $13: 42$ & $\mathrm{C} 2.4$ & & & & & & \\
\hline 20 & Oct. 19,2001 & $16: 48$ & $16: 49$ & $16: 13$ & $16: 30$ & $16: 43$ & $\mathrm{X} 1.6$ & $16: 14$ & $16: 36$ & $18: 49$ & $2 \mathrm{~B}$ & N15 W29 & 9661 \\
\hline
\end{tabular}

\section{References}

Bárta, M., \& Karlický, M. 2001, A\&A, 379, 1045

Chernov, G. P. 2006, Space Sci. Rev., 127, 195

Chernov, G. P., Markeev, A. K., Poquerusse, M., et al. 1998, A\&A, 334, 314

Chernov, G. P., Yan, Y., \& Fu, Q. 2003, A\&A, 406, 1071

Chernov, G. P., Yan, Y., Fu, Q., \& Tan, Ch. 2005, A\&A, 437, 1047

Isliker, H., \& Benz, A. O. 1994, A\&AS, 104, 145

Jiřička, K., Karlický, M., Mészárosová, H., \& Snížek, V. 2001, A\&A, 375, 243

Karlický, M., Bárta, M., Jiřička, K., et al. 2001, A\&A, 375, 638

Krishan, V., Fernandes, F. C. R., Cecatto, J. R., \& Sawant, H. S. 2003, Sol. Phys., 215,147
Kuijpers, J. 1975, Collective Wave-Particle Interactions in Solar Type IV Radio Sources, Utrecht University

Kuznetsov, A. A., \& Tsap, Yu. T. 2007, Sol. Phys., 241, 127

LaBelle, J., Treumann, R. A., Yoon, P. H., \& Karlický, M. 2003, ApJ, 593, 1195 Ledenev, V. G., Karlický, M., Yan, Y., \& Fu, Q. 2001, Sol. Phys., 202, 71

Sawant, H. S., Subramanian, K. R., Faria, C., et al. 2001, Sol. Phys., 200, 167

Sawant, H. S., Fernandes, F. C. R., Cecatto, J. R., et al. 2002, Adv. Space Res., $29,3,349$

Slottje, C. 1981, Atlas of Fine Structures of Dynamic Spectra of Solar Type IV-dm and Some Type II Radio Bursts, Utrecht University

Zheleznyakov, V. V., \& Zlotnik, E. Ya. 1975, Sol. Phys., 43, 431

Zlobec, P., \& Karlický, M. 2007, Sol. Phys., 246, 419 